\title{
Lunar system constraints on the modified theories of gravity
}

\author{
Qasem Exirifard \\ Institute for Research in Fundamental Sciences (IPM), \\ Tehran, Iran \\ E-mail: exir@theory.ipm.ac.ir
}

\begin{abstract}
The MOND paradigm to the missing mass problem requires introducing a functional that is to be identified through observations and experiments. We consider the AQUAL theory as a realization of the MOND. We show that the accurate value of the Earth GM measured by the Lunar Laser Ranging measurements and that by various artificial Earth satellites, including the accurate tracking of the LAGEOS satellites, constrain this functional such that some of the chosen/proposed functional are refuted.
\end{abstract}




\section{Contents}

1 Introduction $\quad 1$

2 A Brief Review of MOND 2

3 MOND Effects on the Lunar Laser Ranging Measurements 3

4 The Lunar System Constraint $\quad 6$

5 Conclusions $\quad 8$

\section{Introduction}

The missing mass problem in galaxies can be resolved either by the Modified Newtonian Dynamics (MOND), or the Modified Gravity (MOG) or the dark matter hypothesis. The first two paradigms require introducing a functional that interpolates the Newtonian regime to the MOND regime. This functional is to be identified by examining the data.

We note that the accurate value of the mass ratio of the Sun/(Earth+Moon) from the Lunar Laser Ranging combined with the Solar GM and the lunar GM from lunar orbiting spacecrafts [8] yields the effective gravitational mass of the Earth in an Earth-centered reference frame with the precision of one part in $10^{8}$ :

$$
G M_{E a r t h}^{L L R}\left(r_{L D}\right)=398600.443 \pm 0.004 \frac{\mathrm{km}^{3}}{\mathrm{~s}^{2}}
$$

where $r_{L D}$ represents the Lunar distance: the average distance between the Earth and Moon. The effective gravitational mass is defined to be the gravitational field multiplied by $r^{2}$ where $r$ is distance from the center of the earth. The effective gravitational mass of the Earth has also been measured by various artificial Earth satellites, including the accurate tracking of the LAGEOS satellites orbiting the Earth in nearly circular orbits with semimajor axes about twice the radius of the Earth [9]:

$$
G M_{\text {Earth }}^{L A G E S}\left(2 r_{\text {Earth }}\right)=398600.4419 \pm 0.0002 \frac{\mathrm{km}^{3}}{\mathrm{~s}^{2}}
$$

where $r_{\text {Earth }}$ stands for the radius of the Earth.

In this paper, we study the earth Lunar system constraints on the interpolation function. In section 2, we first briefly review the MOND paradigm. We present the choices for the interpolating function. The section 3 proves that a relativistic version of MOND does not affect the null geodesics at the precision of the Lunar Laser Ranging measurements. The leading effect of MOND is changing the ratio of the 'Earth-Moon distance' to the 'LAGEOS satellites-Earth distance'. In section 4, we translate the consistency of (1.1) and (1.2) to the first Lunar system constraint on the MOND. We show that the Lunar system constraints refute some of the choices of the interpolating function. 


\section{A Brief Review of MOND}

MOND [1] alters the Newton's second law of dynamics to

$$
F=m f\left(\frac{|a|}{a_{0}}\right) \vec{a},
$$

wherein $F$ is the total force exerted on the particle and [2]

$$
a_{0}=1.0 \times 10^{-10} \frac{m}{s^{2}} .
$$

and $f(x)$ is a function possessing the following asymptotical behaviors

$$
f(x)=\left\{\begin{array}{l}
1, x \gg 1 \\
x, x \leq 1
\end{array} .\right.
$$

MOND is to be applied in a frame inertial with respect to the frame wherein the CMB background is isotropic. The second law of Newtonian dynamic has been tested in a lab on the Earth surface down to the acceleration of $10^{-11} \frac{\mathrm{m}}{\mathrm{s}^{2}}$ [3], and $10^{-14} \frac{\mathrm{m}}{\mathrm{s}^{2}}$ [4]. No deviation has been observed. However, these experiments are not performed in a frame inertial with respect to the CMB frame. They are perfumed in an accelerating frame with respect to the CMB frame: the Earth. So they imply no conclusive constraint on the MOND.

The second approach is theories of modified gravity. We consider the AQUAL (aquadratic Lagrangian theory) model [6]. AQUAL alters the Newtonian gravitational potential equation

$$
\nabla^{i} \nabla_{i} \Phi_{N}=4 \pi G \rho
$$

to

$$
\nabla^{i}\left(\mu\left(\frac{|\nabla \Phi|}{a_{0}}\right) \nabla_{i} \Phi\right)=4 \pi G \rho
$$

where $\mu$ is a functional of the gravitational field strength, and $a_{0}$ is given by (2.2). The asymptotic behavior of $\mu$ is required to coincide to that of $f$ :

$$
\mu(x)=\left\{\begin{array}{l}
1, x \gg 1 \\
x, x \leq 1
\end{array}\right.
$$

Ref. [5] reports that the Newtonian law of gravitation is true at a gravitational acceleration at order of $a_{0}$ in Earth. However, this experiment is performed in the presence of the gravitational field of the Earth. So it implies no conclusive constraint on the AQUAL model. Notice that the AQUAL model fixes only the asymptotic behavior of the functional $\mu$. Various functionals possessing these asymptotic behaviors have been suggested for $\mu$. We consider all the 
families of the suggestions reviewed in [7]:

$$
\begin{aligned}
\mu_{1}(x) & =\frac{\left(1+4 x^{2}\right)^{1 / 2}-1}{2 x}, \\
\mu_{2}(x) & =1-(1+x / 3)^{-3}, \\
\mu_{\alpha}(x) & =\frac{2 x}{1+(2-\alpha) x+\left[(1-\alpha x)^{2}+4 x\right]^{1 / 2}}, \\
\mu_{n}(x) & =\frac{x}{\left(1+x^{n}\right)^{1 / n}}, \\
\nu_{n}(y) & =\left[\frac{1+\left(1+4 y^{-n}\right)^{1 / 2}}{2}\right]^{1 / n}, \\
\nu_{\beta}(y) & =\left(1-e^{-y}\right)^{-1 / 2}+\beta e^{-y}, \\
\nu_{\gamma}(y) & =\left(1-e^{-y^{\gamma / 2}}\right)^{-1 / \gamma}+\left(1-\gamma^{-1}\right) e^{-y^{\gamma / 2}}, \\
\nu_{\delta}(y) & =\left(1-e^{-y^{\delta / 2}}\right)^{-1 / \delta},
\end{aligned}
$$

where

$$
\nu(y)=\frac{1}{\mu(x)},
$$

where

$$
y=x \mu(x) .
$$

Note that $\mu_{1}, \mu_{2}, \mu_{\alpha}, \mu_{n}$ and $\nu_{n}$ connect the MOND regime to the newtonian regime by a power law interpolating function. $\nu_{\beta}, \nu_{\gamma}$ and $\nu_{\delta}$ connect these regimes by an exponentially suppressed interpolating function. We expect that studying the gravity in the Newtonian regime constrains $\mu_{1}, \mu_{2}, \mu_{\alpha}, \mu_{n}$ and $\nu_{n}$. To constrain $\nu_{\beta}, \nu_{\gamma}$ and $\nu_{\delta}$ one should study the MONDian regime of the theory.

\section{MOND Effects on the Lunar Laser Ranging Measurements}

The relativistic versions of MOND attribute the MOND effects to the physical metric that particles feel. They assume that the metric is not what is predicted by the Einstein gravity. Let the space-time geometry around the Earth coincides to that predicted by the Einstein gravity:

$$
d s^{2}=-g_{\mu \nu} d x^{\mu} d x^{\nu} .
$$

A modified theory of gravity adds a correction to the physical metric near the Earth:

$$
d s^{2}=g_{\mu \nu}^{p h y} d x^{\mu} d x^{\nu}=\left(g_{\mu \nu}+g_{\mu \nu}^{(m)}\right) d x^{\mu} d x^{\nu},
$$

where $g_{\mu \nu}^{(m)}$ is the deviation from the prediction of the Einstein theory of gravity.

The leading metric predicted by the Einstein theory can be approximate to the Schwarszchild metric:

$$
\begin{aligned}
g_{\mu \nu} & =g_{\mu \nu}^{(0)}+\epsilon g_{\mu \nu}^{(1)}+\cdots, \\
g_{\mu \nu}^{(0)} d x^{\mu} d x^{\nu} & =\left(1-\frac{r_{h}}{r}\right) c^{2} d t^{2}+\frac{d r^{2}}{1-\frac{r_{h}}{r}}+r^{2} d \Omega^{2}, \\
r_{h} & =\frac{2 G M_{\oplus}}{c^{2}},
\end{aligned}
$$


where the $\epsilon$ terms are due to inhomogeneity of the Earth, and also encode the contribution of the Sun and other objects. We call these terms the $\epsilon$ terms. We assume that the $\epsilon$ terms are sub-leading. In other words the effect of the external gravitational field is sub leading too. We, further, postulate that

$$
g_{\mu \nu}^{(m)}=g_{\mu \nu}^{(m)(0)}+\epsilon g_{\mu \nu}^{(m)(1)}+\cdots,
$$

In other words the deviation from the Einstein prediction inherits the $\epsilon$ expansion series.

Near the Earth the leading term is the Schwarszchild metric. We truncate the series in $\epsilon$ expansion of the metric to the leading term. This implies the truncation of the $\epsilon$ expansion of $g_{\mu \nu}^{(m)}$ to the leading term. The $\epsilon$ expansion in $g_{\mu \nu}^{p h y}$ is not needed for the leading approximation. In this approximation, $g_{\mu \nu}$ possesses spherical symmetry and is stationary. We assume that the corrections due to the considered modified theory of the gravity should respect these symmetries. So within this approximation $g_{\mu \nu}^{(m)}$ should be spherical and stationary:

$$
\begin{aligned}
d s^{2} & =\left(g_{\mu \nu}+g_{\mu \nu}^{(m)}\right) d x^{\mu} d x^{\nu} \\
& =-\left(1-\frac{r_{h}}{r}+g_{t t}^{(m)(0)}\right) c^{2} d t^{2}+\left(\frac{1}{1-\frac{r_{h}}{r}}+g_{r r}^{(m)(0)}\right) d r^{2}+r^{2} d \Omega^{2}+O(\epsilon) .
\end{aligned}
$$

The AQUAL theory only tells us what $g_{t t}^{(m)(0)}$ is. Knowing $g_{t t}^{(m)(0)}$ suffices to address the orbits of non-relativistic objects/probes. However, to address the Null geodesics it is necessary to know $g_{r r}^{(m)(0)}$. Since lasers are used to perform accurate tracking of the Moon and the LAGEOS satellites we must be cautious. We, therefore, estimate the contribution of the modified gravity on measuring long distances by lasers. We prove that this contribution can be consistently neglected at the precision required in this work.

The general solution to the AQUAL model can be expressed in term of the corresponding Newtonian gravitational potential

$$
\mu\left(\frac{|\nabla \Phi|}{a_{0}}\right) \nabla \Phi=\nabla \Phi_{N}+\nabla \times \vec{h}
$$

where $\vec{h}$ is identified by

$$
0=\nabla \times \nabla \Phi=\nabla \times\left(\frac{\nabla \Phi_{N}+\nabla \times \vec{h}}{\mu\left(\frac{|\nabla \Phi|}{a_{0}}\right)}\right) .
$$

In the two-body approximation to the Earth-Moon system, considering the fact that the mass of Moon is much smaller than the Earth's mass, and higher gravitational moments can be neglected, the Newtonian gravitational field around the earth is spherical. This in turn implies that $\vec{h}$ is vanishing for the Earth-Moon system. So the gravitational acceleration near the earth, in the AQUAL model holds

$$
\mu\left(\frac{|\nabla \Phi|}{a_{0}}\right) \nabla \Phi=\nabla \Phi_{N} .
$$

Since near the Earth it holds $\mu \approx 1$ then (3.10) can be perturbatively solved for $\nabla \Phi$

$$
\nabla \Phi \approx \frac{1}{\mu\left(\frac{\left|\nabla \Phi_{N}\right|}{a_{0}}\right)} \nabla \Phi_{N} .
$$


The epsilon expansion in (3.6) provides a sub leading correction to (3.11). Utilizing $\nabla \Phi_{N}=$ $\hat{r} \frac{G M_{\oplus}}{r^{2}}$ results

$$
\nabla \Phi \approx \frac{\hat{r}}{\mu\left(\frac{G M_{\oplus}}{a_{0} r^{2}}\right)} \frac{G M_{\oplus}}{r^{2}} .
$$

Recalling that $\partial_{r} \Phi=-\frac{1}{2} \frac{d g_{t t}}{d r}$ and $g_{t t}=c^{2}\left(1-\frac{r_{h}}{r}+g_{t t}^{(m)}\right)$ then results

$$
g_{t t}^{(m)(0)}(r)-g_{t t}^{(m)(0)}\left(r_{\oplus}\right)=\left(\frac{G M_{\oplus} a_{0}}{c^{4}}\right)^{\frac{1}{2}} \int_{x_{\oplus}}^{x_{r}} \frac{d x}{\sqrt{x}}\left(\frac{1}{\mu(x)}-1\right)
$$

where

$$
x=\frac{G M_{\oplus}}{a_{0} r^{2}},
$$

and $r_{\oplus}$ is the radius of the Earth. We choose the unites of time and space such that

$$
g_{t t}^{(m)(0)}\left(r_{\oplus}\right)=0 .
$$

We are interested to know $g_{t t}^{(m)(0)}(r)$ for $r_{\oplus}<r<r_{L D}$ where $r_{L D}$ is the lunar distance. In this regime

$$
\begin{aligned}
& x_{L D}=\frac{G M_{\oplus}}{a_{0} r_{L D}^{2}}=2.7 \times 10^{7}, \\
& x_{r_{\oplus}}=\frac{G M_{\oplus}}{a_{0} r_{\oplus}^{2}}=9.6 \times 10^{10}, \\
& x_{L D}<x_{r}<x_{r_{\oplus}},
\end{aligned}
$$

so we can expand $\mu(x)$ in large $r$ and keep the leading and the first sub leading terms:

$$
\begin{aligned}
& \mu_{1}(x) \approx 1-\frac{1}{2 x}, \\
& \mu_{2}(x) \approx 1-\frac{27}{x^{3}}, \\
& \mu_{\alpha}(x) \approx 1-\frac{1}{\alpha x}, \\
& \mu_{n}(x) \approx 1-\frac{1}{n x^{n}} .
\end{aligned}
$$

Eq. (2.6) and eq. (2.9) at very large $x$ implies that

$$
y_{r} \approx x_{r} \rightarrow \nu\left(x_{r}\right) \approx \frac{1}{\mu\left(x_{r}\right)},
$$

which in turn yields:

$$
\begin{aligned}
& \tilde{\mu}_{n}(x) \approx \frac{1}{\nu_{n}(x)}=1-\frac{1}{n x^{n}}, \\
& \tilde{\mu}_{\beta}(x) \approx \frac{1}{\nu_{\beta}(x)}=1-(1 / 2+\beta) e^{-x}, \\
& \tilde{\mu}_{\gamma}(x) \approx \frac{1}{\nu_{\gamma}(x)}=1-e^{-x^{\frac{\gamma}{2}}}, \\
& \tilde{\mu}_{\delta}(x) \approx \frac{1}{\nu_{\delta}(x)}=1-\frac{1}{\delta} e^{-x^{\frac{\delta}{2}}} .
\end{aligned}
$$


For all the realistic range of the parameters in the choices of (3.19) and (3.21), one finds that

$$
\int_{r_{\oplus}}^{x_{r}} \frac{d x}{\sqrt{x}}\left(\frac{1}{\mu(x)}-1\right) \ll 1
$$

This implies that

$$
\left|g_{t t}^{(m)(0)}(r)\right| \ll\left(\frac{G M_{\oplus} a_{0}}{c^{4}}\right)^{\frac{1}{2}} \rightarrow\left|g_{t t}^{(m)(0)}(r)\right| \ll 2.21 \times 10^{-15} .
$$

We assume that

$$
\left|g_{r r}^{(m)(0)}(r)\right| \approx\left|g_{t t}^{(m)(0)}(r)\right| \ll 2.21 \times 10^{-15} .
$$

We notice that $g_{r r}^{(m)(0)}(r)$ and $g_{t t}^{(m)(0)}(r)$ contribute to measuring the distance of the EarthMoon at the order of

$$
\Delta L=r_{L D}\left|g_{r r}^{(m)(0)}(r)\right| \ll 10^{-6} \text { meters }
$$

wherein the lunar distance (LD) is set to 384,400 kilometers. Notice that adding the $\epsilon$ expansion (3.6) will not significantly change $\Delta L$. The LLR and LAGEOS satellites identify the center of the mass of the Earth with the precision of centimeters. Therefore, the deviation of the geometry from that predicted from Einstein general relativity can be neglected.

The gravitational field of the Earth, the Sun, the Moon and other planets can cancel each other in a spacial window of order few centimeters. These windows are called the MOND windows. Ref. [12] reports that the size of MOND window at the gravitational saddle points of the Moon and the Earth is about few centimeters. However, identifying the exact time and place of the Earth-Moon system requires taking into account the higher gravitational moments of the Earth and Moon $[13,14]$. The light ray that is used to measure the Moon's distance may occasionally pass through this window. The gravitational field strength inside this window is about and less than $a_{0}$, and its length is about $10 \mathrm{~cm}$. From a simple dimensional analysis one can estimate the change of the metric due to the MOND theory inside the MOND to $p_{M O N D}=\frac{1}{c^{2}} a_{0} * 10 \mathrm{~cm}=10^{-28}$. This change occurs in about $10 \mathrm{~cm}$. So it induces about $10^{-29} \mathrm{~m}$ change in the path of light. The current precision of measuring the Earth-Moon distance is many orders of magnitude larger than $p_{M O N D}{ }^{1}$ The LLR and LAGEOS data we will use in section (4), are also averaged over a long period of time. Therefore occasional passage through the MOND window will not affect them.

\section{The Lunar System Constraint}

In the AQUAL theory it is the eq. (3.11) that represents the gravitational field around the Earth. Now note that (1.1) represents $r^{2} \nabla \Phi$ at the Lunar distance:

$$
\frac{G M_{\oplus}}{\mu\left(\frac{G M_{\oplus}}{a_{0} r_{L D}^{2}}\right)}=398600.443 \pm 0.004 \frac{\mathrm{km}^{3}}{\mathrm{~s}^{2}},
$$

while (1.2) represents $r^{2} \nabla \Phi$ at $r=2 r_{\oplus}$ :

$$
\frac{G M_{\oplus}}{\mu\left(\frac{G M_{\oplus}}{4 a_{0} r_{\oplus}^{2}}\right)}=398600.4419 \pm 0.0002 \frac{\mathrm{km}^{3}}{\mathrm{~s}^{2}},
$$

\footnotetext{
${ }^{1}$ Observing pulsars through the gravitational saddle point (MOND's window) of the Sun-Jupiter system -whose size is about $100 \mathrm{~km}$ - has a better chance to empirically constrain the interaction of light with the physics of the MOND windows.
} 
Consistency between (4.1) and (4.2) then demands that

$$
\left|\frac{1}{\mu\left(x_{L D}\right)}-\frac{1}{\mu\left(x_{2 r_{\oplus}}\right)}\right|<10^{-8},
$$

where $x_{L D}$ is given in (3.16) and

$$
x_{2 r_{\oplus}}=\frac{G M_{\oplus}}{4 a_{0} r_{\oplus}^{2}}=2.4 \times 10^{10},
$$

wherein the radius of the earth is taken to be 6,371 kilometers.

Eq. (4.3) is the Lunar system constraints on the AQUAL functional. $\mu_{1}$ and $\mu_{2}$ hold

$$
\begin{aligned}
& \left|\frac{1}{\mu_{1}\left(x_{L D}\right)}-\frac{1}{\mu_{1}\left(x_{2 r_{\oplus}}\right)}\right|=1.85 \times 10^{-8}, \\
& \left|\frac{1}{\mu_{2}\left(x_{L D}\right)}-\frac{1}{\mu_{2}\left(x_{2 r_{\oplus}}\right)}\right|=1.37 \times 10^{-21} .
\end{aligned}
$$

So the choice of $\mu_{1}$ is refuted at the confidence level of 1.85 sigmas while $\mu_{2}$ remains consistent with the Lunar system constraints. Eq. (4.3) also constraints $\alpha$ and $n$ in (2.7c) and (2.7d):

$$
\begin{gathered}
\alpha>3.69954, \\
n>1.07237 .
\end{gathered}
$$

Due to (3.21a), (4.3) implies $n>1.072$ for $(2.7 \mathrm{e})$. Since $\nu_{\beta}(y)-1, \nu_{\gamma}(y)-1$ and $\nu_{\delta}(y)-1$ are exponentially suppressed in large y, (4.3) provides no strong constraint on them. Those results, however, can be interpreted as limits on the intermediate-range gravity [15]. Note that [15] has used the same set of data but has applied it to a single choice for the interpolating function. Ref. [7] within its 6.4 section reviews the Solar constraints on $\mu$. Though the Lunar system constraint presented here is weaker than the constraint due to the perihelion precession of the Saturn reported in $[10,11]^{2}$, note that here we have used a different set of data. Furthermore our constraint is directly derived from the MOND effect, and in contrary to $[10,11]$ is not based on to the external field effects.

Let us apply the Lunar system constraint on the standard, the standard and the Bekenstein's choices [16]:

$$
\begin{aligned}
\mu_{\text {standard }} & =\left.\mu_{n}(x)\right|_{n=2}=\frac{x}{\sqrt{1+x^{2}}}, \\
\mu_{\text {simple }} & =\left.\mu_{n}(x)\right|_{n=1}=\frac{x}{1+x}, \\
\mu_{\text {Bekenstein }} & =\left.\mu_{\alpha}(x)\right|_{\alpha=0}=\frac{\sqrt{1+4 x}-1}{\sqrt{1+4 x}+1} .
\end{aligned}
$$

The Lunar system constraint is consistent with the standard choice. It, however, refutes the simple choice with the confidence level of 3.69 sigmas. It refutes the Bekenstein choice at the confidence level of 18600 sigmas.

\footnotetext{
${ }^{2}$ They constrain $(2.7 \mathrm{~d})$ to $n>8$.
} 


\section{Conclusions}

We have translated the GM mass measured by the Lunar Laser ranging measurements and LAGEOS satellites into a constraint on the interpolating function of modified gravities. The Lunar constraint is given in (4.3). We have applied the Lunar system's constraint on all the families of the interpolating function considered in the literature and reviewed in [7]. We have proven that the Lunar system constraint is non-trivial for a set of families. In particular we have proven that the Lunar system constraint refutes the simple interpolating function at the confidence level of the 3.69 sigmas, and completely refutes the Bekenstein choice.

\section{Acknowledgments}

This work was supported by the Institute for Research in Fundamental Sciences (IPM).

\section{References}

[1] M. Milgrom, "A modification of the Newtonian dynamics as a possible alternative to the hidden mass hypothesis", Astrophys. J. 270 (1983) 365; M. Milgrom, "A modification of the Newtonian dynamics: Implications for galaxies", Astrophys. J. 270 (1983) 371.

[2] J. D. Bekenstein, "The modified Newtonian dynamics-MOND-and its implications for new physics", arXiv:astro-ph/0701848; K. G. Begeman, A. H. Broeils and R. H. Sanders, "Extended rotation curves of spiral galaxies: Dark haloes and modified dynamics", Mon. Not. Roy. Astron. Soc. 249 (1991) 523.

[3] A. Abramovici and Z. Vager, "Test Of Newton's Second Law At Small Accelerations," Phys. Rev. D 34 (1986) 3240.

[4] J. H. Gundlach, S. Schlamminger, C. D. Spitzer, K. Y. Choi, B. A. Woodahl, J. J. Coy and E. Fischbach, "Laboratory Test of Newton's Second Law for Small Accelerations," Phys. Rev. Lett. 98 (2007) 150801.

[5] H. Meyer, E. Lohrmann, S. Schubert, W. Bartel, A. Glazov, B. Loehr, C. Niebuhr and E. Wuensch et al., "Test of the Law of Gravitation at small Accelerations," arXiv:1112.0434 [gr-qc].

[6] J. Bekenstein, M. Milgrom, "Does the missing mass problem signal the breakdown of Newtonian gravity", Astrophysical J. 286, (1984) 7.

[7] B. Famaey and S. McGaugh, "Modified Newtonian Dynamics (MOND): Observational Phenomenology and Relativistic Extensions," Living Rev. Rel. 15 (2012) 10 [arXiv:1112.3960 [astro-ph.CO]].

[8] J. O. Dickey, P. L. Bender, J. E. Faller, X. X. Newhall, R. L. Ricklefs, J. G. Ries, P. J. Shelus, C. Viellet, A. L. Whipple, J. R. Wiant, J. G. Williams, C. F. Yoder, "Lunar laser ranging: A continuing legacy of the Apollo program", Science 265 (1994) 482.

[9] J. C. Ries, R. J. Eanes, C. K. Shum, M. M. Watkins, Geophys. Res. Lett. 19 (1992) 529; P. Dunn, M. Torrence, R. Kolenkiewicz, D. Smith, "Earth scale deї£ned by modern satellite ranging observations", Geophy. Res. Lett. 26 (1999) 1489.

[10] L. Blanchet and J. Novak, "Testing MOND in the Solar System, arXiv:1105.5815 [astro-ph.CO].

[11] A. Fienga, J. Laskar, P. Kuchynka, C. Leponcin-Lafitte, H. Manche and M. Gastineau, "Gravity tests with INPOP planetary ephemerides," arXiv:0906.3962 [gr-qc].

[12] P. Galianni, M. Feix, H. Zhao and K. Horne, "Testing quasilinear modified Newtonian dynamics in the Solar System," Phys. Rev. D 86 (2012) 044002 [arXiv:1111.6681 [astro-ph.EP]]. 
[13] A. S. Konopliv, A. B. Binder, L. L. Hood, A. B. Kucinskas, W. L. Sjogren, J. G. Williams "Improved Gravity Field of the Moon from Lunar Prospector," Science, 281 (1998) 5382 : $1476-1480$.

[14] F. G. Lemoine, D. E. Smith, R. Smith, L. Kunz, N. K. Pavlis, S. M. Klosko, D. S. Chinn, M. H. Torrence, R. G. Williamson, C. M. Cox, K. E. Rachlin, Y. M. Wang, E. C. Pavlis, S. C. Kenyon, R. Salman, R. Trimmer and R. H. Rapp, "The NASA GSFC and NIMA Joint Geopotential Model", http://cddis.nasa.gov/926/egm96/getit.html.

[15] G. -y. Li and H. Zhao, "Constraint on intermediate-range gravity from earth-satellite and lunar orbiter measurements, and lunar laser ranging," Int. J. Mod. Phys. D 14 (2005) 1657 [gr-qc/0505090].

[16] H. -S. Zhao and B. Famaey, "Refining mond interpolating function and teves Lagrangian," Astrophys. J. 638 (2006) L9 [astro-ph/0512425]. 